\title{
ARTICLE OPEN Predicting two-dimensional topological phases in Janus materials by substitutional doping in transition metal dichalcogenide monolayers
}

Aniceto B. Maghirang III ${ }^{1}$, Zhi-Quan Huang ${ }^{1}$, Rovi Angelo B. Villaos ${ }^{1}$, Chia-Hsiu Hsu ${ }^{1}$, Liang-Ying Feng ${ }^{1}$, Emmanuel Florido ${ }^{2}$, Hsin Lin ${ }^{3}$, Arun Bansil ${ }^{4}$ and Feng-Chuan Chuang (iD)

Ultrathin Janus two-dimensional (2D) materials are attracting intense interest currently. Substitutional doping of 2D transition metal dichalcogenides (TMDs) is of importance for tuning and possible enhancement of their electronic, physical and chemical properties toward industrial applications. Using systematic first-principles computations, we propose a class of Janus 2D materials based on the monolayers $\mathrm{MX}_{2}(\mathrm{M}=\mathrm{V}, \mathrm{Nb}, \mathrm{Ta}$, Tc, or $\mathrm{Re} ; \mathrm{X}=\mathrm{S}$, Se, or $\mathrm{Te})$ with halogen $(\mathrm{F}, \mathrm{Cl}, \mathrm{Br}$, or I) or pnictogen $(\mathrm{N}, \mathrm{P}, \mathrm{As}, \mathrm{Sb}$, or $\mathrm{Bi})$ substitution. Nontrivial phases are obtained on pnictogen substitution of group VB (V, Nb, or Ta), whereas for group VIIB (Tc or Re), the nontrivial phases are obtained for halogen substitution. Orbital analysis shows that the nontrivial phase is driven by the splitting of $\mathrm{M}-d_{y z}$ and $\mathrm{M}-d_{x z}$ orbitals. Our study demonstrates that the Janus 2D materials have the tunability and suitability for synthesis under various conditions.

npj 2D Materials and Applications (2019)3:35 ; https://doi.org/10.1038/s41699-019-0118-2

\section{INTRODUCTION}

Topological insulators (TIs) have been intensely studied for the past few years since the discovery of graphene due to their interesting electrical, optical, and mechanical properties. Tls are uniquely insulating in the bulk, but support conducting edge and surface states in 2D and 3D, respectively. ${ }^{1-4}$ In particular, twodimensional topological insulators (2D TIs), also known as quantum spin Hall (QSH) insulators, exhibit unique symmetryprotected helical metallic edge states with an insulating interior, which makes these materials especially well-suited for optoelectronic, spintronics, quantum computing, and other applications due to the robustness of their edge states against backscattering. ${ }^{5}$ Numerous theoretical studies have been focused on identifying new materials that can support $2 \mathrm{D} \mathrm{TI^{1,6-9 }}$ or topological crystalline insulator $(\mathrm{TCl})^{10-13}$ phases. Moreover, effects of functionalization $^{7,14-16}$ and substrates ${ }^{16-18}$ have been investigated, although experimental realizations are quite limited so far. ${ }^{19-21}$

In this connection, one of the most promising classes of 2D materials with semiconducting properties are the transition metal dichalcogenides (TMDs) with chemical formula $M X_{2}$, where $M$ is a transition metal atom and $X$ is a chalcogen atom, which exhibit different structural phases such as $1 \mathrm{~T}$ (octahedral) and $2 \mathrm{H} / 3 \mathrm{R}$ (trigonal prismatic). Despite graphene's popularity, the lack of an electronic bandgap in graphene has driven the interest in 2D TMD films as a promising alternative. ${ }^{22,23}$

Recent theoretical ${ }^{24-28}$ and experimental ${ }^{29-35}$ studies show that doping is a viable method for enhancing and tuning electronic and optical properties of TMDs for potential applications. One specific type of doping is substitutional doping, and there have been reports of successful experimental substitution of transition metal $^{29}$ or chalcogen atoms. ${ }^{35}$ Most experimental research has focused on doping $\mathrm{MoS}_{2}$. For example, covalent nitrogen doping of $\mathrm{MoS}_{2}$ was obtained by remote $\mathrm{N}_{2}$ plasma exposure and it reportedly can induce compressive strain on TMDs ${ }^{31}$ (as can other experimental procedures ${ }^{32,33}$ ), and computational results show that structural phase transitions can occur by alloying. ${ }^{28}$

Very recently, ultrathin 2D Janus materials have started to attract attention. In fact, a number of honeycomb-like 2D Janus materials that could harbor topological phases have been predicted. $^{16,17,36-39}$ In addition, in TMD materials, exemplary results have been obtained experimentally where S-Mo-Se crystal structure (also known as Janus SMoSe) was successfully synthesized by well-controlled sulfurization of monolayer $\mathrm{MoSe}_{2}{ }^{35}$ confirming the possibility of chalcogen substitution. Although a number of electronic structure calculations have been performed, substitution studies are sparse and much less explored, ${ }^{40,41}$ indicating the need for the development of other doping strategies. Also, since substitutional doping is relatively at an early stage of research, ${ }^{42}$ exploration of other TMDs that could be doped is highly desirable. To our knowledge, one of the criteria in choosing a dopant is based on the capability to change the total number of electrons (hole doping or electron doping) in a compound. ${ }^{43}$ This suggests that, aside from substituting one chalcogen with another chalcogen, it will be interesting also to consider substitution with group VA (pnictogen) ${ }^{44}$ or group VIIA (halogen). ${ }^{45}$

Among the TMDs, groups VB and VIIB TMDs have odd number of electrons as well as weak magnetism and spin-orbit coupling,

\footnotetext{
'Department of Physics, National Sun Yat-sen University, Kaohsiung, Taiwan; ${ }^{2}$ Institute of Mathematical Sciences and Physics, College of Arts and Sciences, University of the Philippines Los Baños, College, Laguna, Philippines; ${ }^{3}$ Institute of Physics, Academia Sinica, Taipei, Taiwan and ${ }^{4}$ Department of Physics, Northeastern University, Boston, MA 02115, USA

Correspondence: Feng-Chuan Chuang (fchuang@mail.nsysu.edu.tw)

These authors contributed equally: Aniceto B. Maghirang III, Zhi-Quan Huang, Rovi Angelo B. Villaos
}

Received: 28 January 2019 Accepted: 20 August 2019

Published online: 12 September 2019 
and their pristine films are metallic. ${ }^{25,46-49}$ Thus, substitutional doping of VA or VIIA or one-sided $\mathrm{H}$ adsorption, will result in making the system acquire an even number of electrons. Also, pristine ultrathin films of a number of these materials $\left(\mathrm{TaS}_{2}{ }^{50}\right.$ $\mathrm{TaSe}_{2}$, $\mathrm{NbSe}_{2},{ }^{52,53} \mathrm{NbS}_{2}, \mathrm{VS}_{2},{ }^{55} \mathrm{VSe}_{2},{ }^{56} \mathrm{VTe}_{2},{ }^{57} \mathrm{ReS}_{2},{ }^{58,59}$ and $\mathrm{ReSe}_{2}{ }^{60}$ ) have been experimentally synthesized, suggesting that the synthesis of the related Janus 2D materials based on VB and VIIB considered in this study will likely be viable.

So motivated, we have carried out a first-principles study of possible Janus 2D topological insulator phases via halogen $(\mathrm{F}, \mathrm{Cl}$, $\mathrm{Br}$, or $\mathrm{I})$ and pnictogen (N, $\mathrm{P}, \mathrm{As}, \mathrm{Sb}$, or $\mathrm{Bi})$ substitution, including one-sided hydrogen adsorption, of $\mathrm{MX}_{2}(\mathrm{M}=\mathrm{V}, \mathrm{Nb}, \mathrm{Ta}, \mathrm{Tc}$, or Re; $\mathrm{X}=\mathrm{S}$, Se, or Te) films in both $1 \mathrm{~T}$ (octahedral) and $2 \mathrm{H}$ (trigonal prismatic) structures. A total of 294 compounds were examined. Structural phase (from $2 \mathrm{H}$ to $1 \mathrm{~T}$ ) and magnetic property (between non-magnetic and ferromagnetic states) transitions were also observed upon substitutional doping. 2D nontrivial phases were found for pnictogen substitution of $M X_{2}(M=V, N b$, or $T a)$, while the nontrivial phases were found for $M_{2}(M=T c$ or $R e)$ with halogen substitution, and one-sided $\mathrm{H}$ adsorption.

\section{RESULTS}

Figure 1 shows the perspective views of the investigated monolayer $1 \mathrm{~T}$ and $2 \mathrm{H} \mathrm{MX}, \mathrm{MXY}$, and $\mathrm{MX}_{2} \mathrm{H}(\mathrm{M}=$ transition metal, $\mathrm{X}=$ chalcogen, $\mathrm{Y}=$ halogen or pnictogen, $\mathrm{H}=$ Hydrogen) and the related first Brillouin Zone (BZ). The calculated stable structures of $M X_{2}, M X_{2} H$, and $M X Y$ are shown in Table 1. Table 2 gives the calculated topological invariant $Z_{2}$ for the stable structural phase of $\mathrm{MX}_{2}, \mathrm{MX}_{2} \mathrm{H}$, and $\mathrm{MXY}$. Tables 3 and 4 show the calculated topological invariant $Z_{2}$ for the stable structural phase of group VB upon pnictogen substitutional doping and group VIIB upon one hydrogen adsorption and halogen substitutional doping, respectively. In order to unfold the electronic properties of the stable materials, band structures of the $\mathrm{TaS}_{2}$-based films as representative of group VB are shown in Fig. 2, while results for the $\mathrm{ReSe}_{2}-$ based films as representative of group VIIB are shown in Fig. 3. In order to accurately estimate the band gaps and better understand the mechanism underlying various splittings, hybrid-functional based band structures of the selected stable structural phases, including detailed orbital analysis and crystal field splittings are shown in Fig. 4. Illustrations of the topologically protected edge states, and possible synthesis of Janus 2D materials via lithography are presented in Fig. 5.

\section{DISCUSSION}

The structures of the investigated monolayer $\mathrm{MX}_{2}(\mathrm{M}=\mathrm{V}, \mathrm{Nb}, \mathrm{Ta}$, $\mathrm{Tc}$, or Re; $\mathrm{X}=\mathrm{S}, \mathrm{Se}$, or $\mathrm{Te}$ ) are assumed to be in $1 \mathrm{~T}$ or $2 \mathrm{H}$. The perspective views of the atomic structure for both pristine 1T and $2 \mathrm{H}$ are shown in Fig. 1a, d. Next, the structures of Janus TMDs (MXY) in which one halogen or one pnictogen substitutes one chalcogen per unit cell are shown in Fig. 1b, e. Lastly, we consider the case where one $\mathrm{H}$ is directly adsorbed on top of the chalcogen atom as shown in Fig. 1c, f. The surface Brillouin-zones is presented in Fig. 1g.

Optimization of the atomic structures and lattice constants was done using the methods discussed in the Methods section below. Relevant information regarding the optimized structures is summarized in Tables S1-S10 in the Supplementary Materials, and includes details of lattice constants, stable phases, as well as the energetics of the two structures using different magnetic configurations (non-magnetic and ferromagnetic).

Regarding structural stability, Table 1 shows that our result that the structurally stable phase for all the investigated pristine $\mathrm{MX}_{2}$ films is $2 \mathrm{H}$, which is in agreement with other theoretical and experimental findings, ${ }^{25,46-48}$. Subsequently, we performed the substitution of chalcogen atoms with pnictogen atoms (group VA) or halogen atoms (group VIIA) on one side, as well as onehydrogen-adsorption on the chalcogen. Hydrogen adsorption is assumed to have a similar effect to halogen substitution in terms of changing the total number of valence electrons in pristine $M X_{2}$. Consequently, after the aforementioned substitution or hydrogen adsorption, the total number of valence electrons would become even due to the initial odd number in pristine $M X_{2}$. Moreover, numerous studies have shown that the structural phase transition may occur after doping. ${ }^{25,28,32,33}$ Our findings for group VB indeed indicate a structural phase transition from $2 \mathrm{H}$ to $1 \mathrm{~T}$ for all the $M X Y_{\text {pnic }}$ films, whereas the structures after halogen substitution or hydrogen adsorption are found to stay $2 \mathrm{H}$. On the other hand, our findings for group VIIB indicate a structural phase transition from $2 \mathrm{H}$ to $1 \mathrm{~T}$ for all the $M X Y_{\text {hal }}$ or $\mathrm{MX}_{2} \mathrm{H}$ films, while for pnictogen substitution, the structures stay $2 \mathrm{H}$. All these results are summarized in Table 1.

To corroborate the structural stability of the selected stable MXY films, the corresponding phonon spectra were calculated. A total of nine phonon bands were found in each selected case, with three acoustic and six optical branches, as shown in Figs. S1-S4. The group VIIB MXY films exhibit negative frequency (Fig. S4a and b) and are thus, structurally unstable. In contrast, these negative
$1 T$

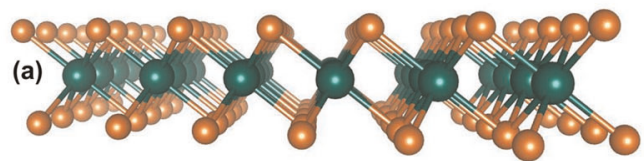

(b)
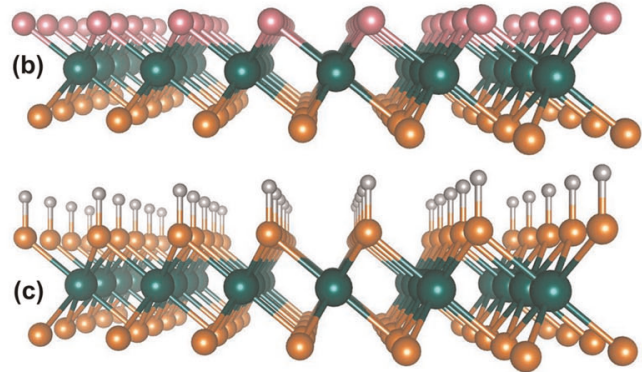

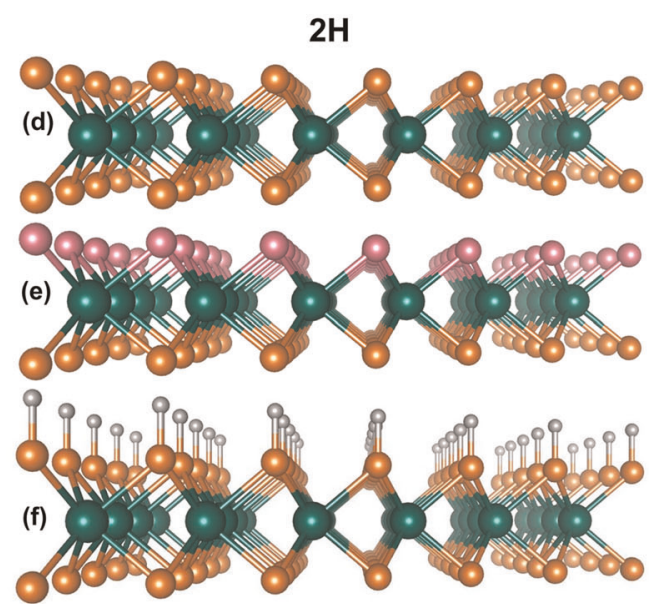

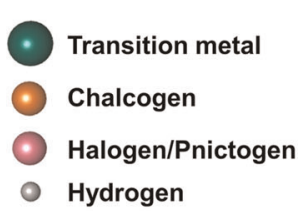

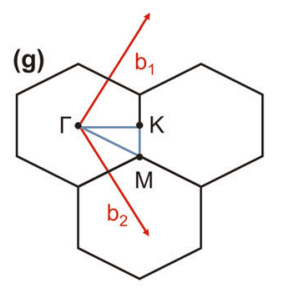

Fig. 1 Perspective views of monolayer $1 \mathrm{~T}$ and $2 \mathrm{H} \mathrm{MX}$ and $M X Y$ films ( $M=$ transition metal, $X=$ chalcogen, $Y=$ halogen or pnictogen). $\mathbf{a}$, $\mathbf{d}$ Pristine $\mathrm{MX}_{2}$. b. e Structures with one halogen, or one pnictogen, substituting one chalcogen per unit cell. c, $\mathbf{f}$ Structures with one adsorbed hydrogen atom on a chalcogen atom. g 2D Brillouin-zones with the specific high symmetry points labeled 
Table 1. Calculated stable structures of $M_{2}, M X_{2} H$, and $M X Y$

\begin{tabular}{llllll}
\hline & $V$ & $\mathrm{Nb}$ & $\mathrm{Ta}$ & $\mathrm{Tc}$ & $\mathrm{Re}$ \\
\hline $\mathrm{X}_{2}$ & $2 \mathrm{H}$ & $2 \mathrm{H}$ & $2 \mathrm{H}$ & $2 \mathrm{H}$ & $2 \mathrm{H}$ \\
$\mathrm{X}_{2} \mathrm{H}$ & $2 \mathrm{H}$ & $2 \mathrm{H}$ & $2 \mathrm{H}$ & $1 \mathrm{~T}$ & $1 \mathrm{~T}$ \\
$M_{X Y_{\text {hal }}}$ & $2 \mathrm{H}$ & $2 \mathrm{H}$ & $2 \mathrm{H}$ & $1 \mathrm{~T}$ & $1 \mathrm{~T}$ \\
$M \mathrm{Y}_{\text {pnic }}$ & $1 \mathrm{~T}$ & $1 \mathrm{~T}$ & $1 \mathrm{~T}$ & $2 \mathrm{H}$ & $2 \mathrm{H}$ \\
\hline
\end{tabular}

Table 2. Calculated topological invariant $\mathrm{Z}_{2}$ for the stable structural phases of $\mathrm{MX}_{2}, \mathrm{MX}_{2} \mathrm{H}$, and MXY

\begin{tabular}{llllll}
\hline & $V$ & $N b$ & $T a$ & $T c$ & $R e$ \\
\hline$X_{2}$ & $M$ & $M$ & $M$ & $M$ & $M$ \\
$X_{2} H$ & 0 & 0 & 0 & Refer to Table 4 & \\
$M X Y_{\text {hal }}$ & 0 & 0 & 0 & & 0 \\
$M X Y_{\text {pnic }}$ & Refer to Table 3 & & 0 & \\
\hline$Z_{2}=0$ indicates the trivial phase, whereas $Z_{2}=1$ is for the nontrivial phase. \\
$Z_{2}$ invariant is not applicable to a metal, and for this reason, metallic \\
systems are denoted by $M$
\end{tabular}

Table 3. Calculated topological invariant $Z_{2}$ for the stable structural phase of group VB upon pnictogen substitutional doping

\begin{tabular}{|c|c|c|c|c|c|c|c|c|c|c|c|c|c|c|}
\hline & \multicolumn{4}{|l|}{$X$} & & \multicolumn{4}{|l|}{$X$} & & \multicolumn{4}{|l|}{$X$} \\
\hline & V & $S$ & $\mathrm{Se}$ & $\mathrm{Te}$ & & $\mathrm{Nb}$ & $S$ & $\mathrm{Se}$ & $\mathrm{Te}$ & & $\mathrm{Ta}$ & $S$ & $\mathrm{Se}$ & $\mathrm{Te}$ \\
\hline \multirow[t]{4}{*}{$Y$} & P & 1 & $M$ & 0 & $Y$ & $P$ & 0 & 0 & 0 & $Y$ & P & 0 & 0 & 0 \\
\hline & As & $M$ & 1 & $M$ & & As & 1 & $\mathrm{M}$ & 0 & & As & $M$ & $M$ & 0 \\
\hline & $\mathrm{Sb}$ & $M$ & 1 & 1 & & $\mathrm{Sb}$ & 1 & 1 & 1 & & $\mathrm{Sb}$ & 1 & 1 & 0 \\
\hline & $\mathrm{Bi}$ & 1 & 1 & 1 & & $\mathrm{Bi}$ & 1 & 1 & 1 & & $\mathrm{Bi}$ & 1 & 1 & 1 \\
\hline
\end{tabular}

$Z_{2}=0$ indicates the trivial phase, whereas $Z_{2}=1$ is for the nontrivial phase. $\mathrm{Z}_{2}$ invariant is not applicable to a metal, and for this reason, metallic systems are denoted by $\mathrm{M}$

Table 4. Calculated topological invariant $Z_{2}$ for the stable structural phases of group VIIB upon one hydrogen adsorption and halogen substitutional doping

\begin{tabular}{|c|c|c|c|c|c|c|c|c|c|}
\hline & \multicolumn{4}{|l|}{$x$} & & \multicolumn{4}{|l|}{$x$} \\
\hline & Tc & $S$ & Se & Te & & $\operatorname{Re}$ & $S$ & $\mathrm{Se}$ & Te \\
\hline$x$ & w/H & $C=-2$ & $C=-1$ & $C=-2$ & $X$ & $\mathrm{w} / \mathrm{H}$ & 1 & $C=-2$ & 1 \\
\hline \multirow[t]{4}{*}{ Y } & $F$ & 0 & 0 & 1 & Y & $\mathrm{F}$ & 0 & 1 & 1 \\
\hline & $\mathrm{Cl}$ & M & 1 & $M$ & & $\mathrm{Cl}$ & 0 & 1 & $C=-2$ \\
\hline & $\mathrm{Br}$ & 0 & 0 & $C=-1$ & & $\mathrm{Br}$ & 1 & 1 & $C=-2$ \\
\hline & I & M & M & $C=-1$ & & I & $C=0$ & 0 & $C=-2$ \\
\hline
\end{tabular}

$Z_{2}=0$ indicates the trivial phase, whereas $Z_{2}=1$ is for nontrivial phases of the non-magnetic films. Chern number, on the other hand, indicates the trivial phase $(C=0)$ and nontrivial phase $(C \neq 0)$ for the ferromagnetic cases. $Z_{2}$ and $C$ invariants not applicable to a metal, and for this reason, metallic systems are denoted by $M$

frequency modes did not occur in the group VB MXY films as shown in Figs. S1-S3, indicating their structural stability and viability of experimental synthesis. Together with the phonon spectra, the formation energies were also calculated to further check their stability. The formation energies and energy differences shown in Table S11 are consistent with the results of phonon spectra. Group VB MXY films exhibit lower energies compared to the corresponding pristine phases with a positive energy difference, indicating structural stability. On the other hand, group VIIB MXY have higher energy compared to their pristine counterparts with a negative energy difference, indicating structural instability. We have also carried out first-principles molecular dynamics (MD) simulations using the NVT ensemble for a $3 \times$ 3 superlattice at $500 \mathrm{~K}$ as shown in Figs. S5-S9. Group VB MXY structures are found to be dynamically stable even at elevated temperature, while this is not the case for the group VII MXY structures. The MD results are thus also consistent with the results of phonon spectra.

To further investigate the band topologies of the computed stable compounds, we follow the method of Refs. ${ }^{61,62}$ for calculating the $Z_{2}$ topological invariant in non-magnetic cases or Chern number in ferromagnetic cases. We have computed the $Z_{2}$ or the Chern number of all the systems we investigated for completeness, including the unstable phase, see Tables S1-S5 under Supplementary Materials.

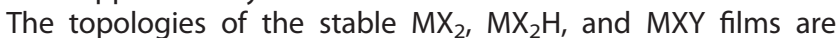
summarized in Tables 2-4. As shown in Table 2, the pristine $\mathrm{MX}_{2}$ films exhibit a metallic state due to their half-filled band. Further shown in Table 2 are the $Z_{2}$ invariants of group VB MXY $\mathrm{MX}_{2} \mathrm{H}$ as well as group VIIB MXY pnic films, which are found to be zero corresponding to the trivial phase. These trivial metallic phases were also found in several group VB MXY pnic films. But, importantly, we found a total of nineteen compounds belonging to the VXY, NbXY, and TaXY film clasees, with 7, 7, and 5 nontrivial phases, $Z_{2}=1$, respectively. Notably, the nontrivial phases we have found are mostly generated through the substitution by relatively heavy atoms such as $\mathrm{Sb}$ and $\mathrm{Bi}$. The Table 3 summarizes our results. Moving to group VIIB, several metallic states were only found in halogen substituted TcXY films. A total of 18 compounds in $\mathrm{TcXY}, \mathrm{TcX}{ }_{2} \mathrm{H}, \mathrm{ReXY}$, and $\mathrm{ReX}_{2} \mathrm{H}$ films exhibited non-trivial phases, see Table 4. We can see that upon pnictogen substitution of group VB, several nontrivial phases were induced, whereas the occurrence of nontrivial phases in group VIIB arose upon halogen substitution or one-sided $\mathrm{H}$ adsorption. These results support the notion that the effects of halogen substitution and one-sided $\mathrm{H}$ adsorption are similar in that both of theses dopings induce the same structural phase transition between the trivial or nontrivial phases.

To gain further insight, we explore the band structures of $\mathrm{TaS}_{2}-$ based films as representative of group VB. As shown in Fig. 2a and e, our band structures of the pristine $\mathrm{TaS}_{2}$ films are in agreement with previous studies. ${ }^{47} \mathrm{TaS}_{2}$ has an odd number of electrons, so that the Fermi level crosses the highest occupied band. By substituting one chalcogen with one halogen, one extra electron is introduced, which could also be done by adsorbing adding one hydrogen on $\mathrm{TaS}_{2}$. The effects of halogen substitution and onesided hydrogenation on the pristine $\mathrm{TaS}_{2}$ are indeed found to be similar, both inducing a downward shift in the band structures resulting in a trivial phase as shown in Fig. $2 \mathrm{~b}, \mathrm{c}, \mathrm{f}$, and $\mathrm{g}$. This is true for all group VB MXY hal and $\mathrm{MX}_{2} \mathrm{H}$ films.

Referring to Table 1, in contrast to halogen substitutions and hydrogen adsorption, we find that pnictogen substitution induces a structural transition from $2 \mathrm{H}$ to $1 \mathrm{~T}$ in group $\mathrm{VB}$, and for this reason, the $1 \mathrm{~T}$ band structures are shown in Fig. $2 \mathrm{~d}$, and $\mathrm{h}$. Contrary to halogen substitution and one-sided hydrogenation, pnictogen substitution results in the loss of one electron. In the case of TaSBi film (without SOC), we see from Fig. $2 \mathrm{~d}$ that HOMO and LUMO levels touch at $\Gamma$. When the SOC is included, a $108 \mathrm{meV}$ gap opens at the $\Gamma$ point. The calculated topological invariant of $1 \mathrm{~T} \mathrm{TaSBi}$ film is $Z_{2}=1$ (Table 3 ), so that this film harbors a nontrivial phase.

We turn now to discuss in-depth stable $\mathrm{ReSe}_{2}$ films as representative of group VIIB. Referring to Table 1, we see that 

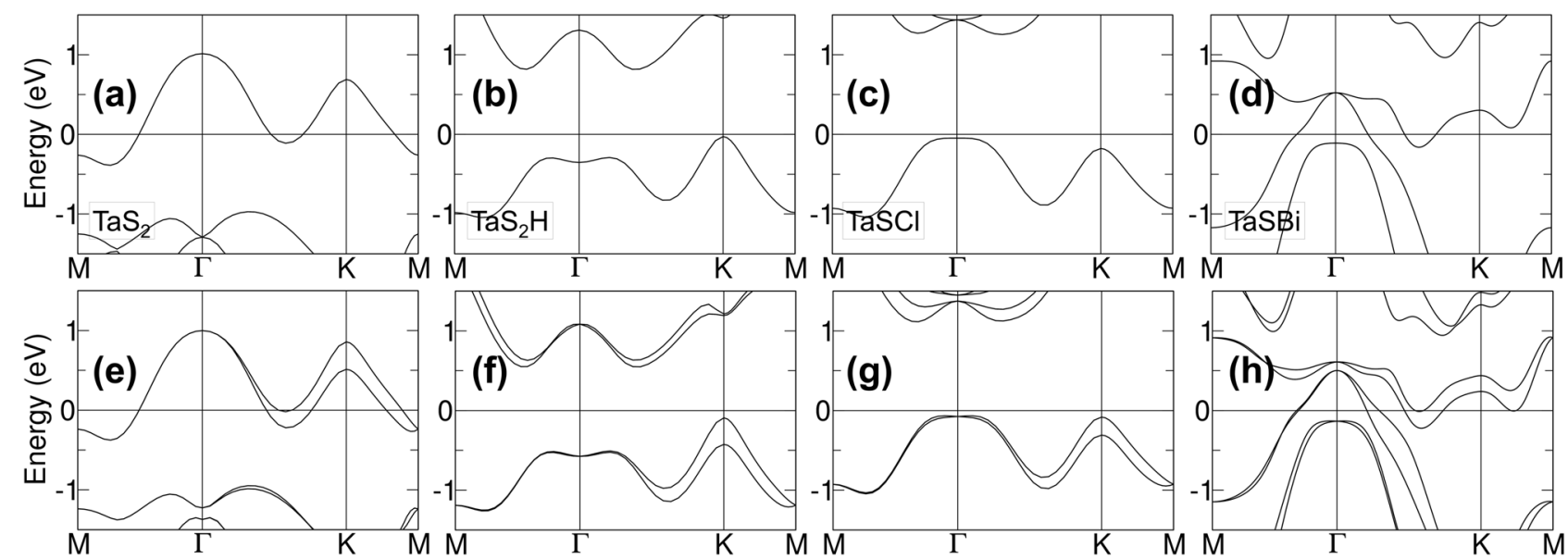

Fig. 2 Band structures of the stable structural phases without SOC (top) and with $\mathrm{SOC}$ (bottom). a, e Monolayer pristine TaS $\mathrm{f}_{2}$ film in the $2 \mathrm{H}$ structure. $\mathbf{b}, \mathbf{f}$ One adsorbed hydrogen on $\mathrm{TaS}_{2}$ in the $2 \mathrm{H}$ structure. $\mathbf{c}, \mathbf{g}$ One $\mathrm{Cl}$ atom substituting a $\mathrm{S}$ atom in the $2 \mathrm{H}$ structure. $\mathbf{d}, \mathbf{h}$ One $\mathrm{Bi}$ atom substituting a $\mathrm{S}$ atom in the $1 \mathrm{~T}$ structure
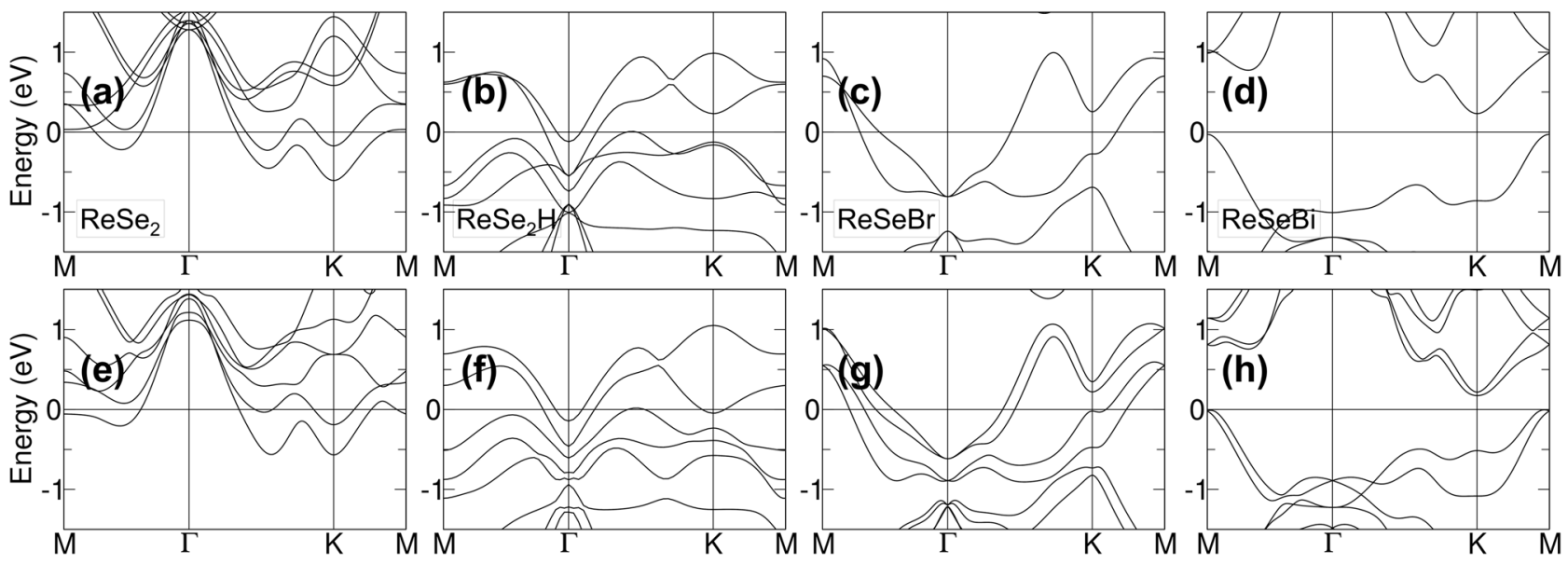

Fig. 3 Band structures of the stable structural phases without SOC (top) and with SOC (bottom). a, e Monolayer pristine ReSe 2 in $2 \mathrm{H}$ structure. $\mathbf{b}, \mathbf{f}$ One adsorbed hydrogen on $\mathrm{ReSe}_{2}$ film in the 1T structure. $\mathbf{c}, \mathbf{g}$ One $\mathrm{Br}$ atom substituting a Se atom in the 1T structure. $\mathbf{d}, \mathbf{h}$ One $\mathrm{Bi}$ atom substituting a $\mathrm{Se}$ atom in the $2 \mathrm{H}$ structure

halogen substitutions and hydrogen adsorptions can induce a structural phase transition from $2 \mathrm{H}$ to $1 \mathrm{~T}$ in group VIIB, and here again for this reason, 1T band structures are presented in Fig. 3b, c, $f$, and g. In Fig. 3a and e, our band structures for the pristine $\mathrm{ReSe}_{2}$ film are in agreement with previous studies. ${ }^{25}$ Similar to the case of $\mathrm{TaS}_{2}$, the ReSe ${ }_{2}$ film has an odd number of electrons, and thus the Fermi level crosses the highest occupied band as expected. A splitting driven by the SOC is evident in Fig. $3 f$ and $g$ for one hydrogen adsorption and halogen substitution, respectively, and respective band gaps at $\Gamma$ of 145 and $274 \mathrm{meV}$. The calculated

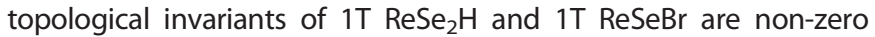
Chern number and $Z_{2}=1$, respectively, indicating a nontrivial phase. The effect of halogen substitution and one-sided hydrogenation on the pristine $\mathrm{ReSe}_{2}$ film are found to be similar in that they induce a downward shift in the band structure and result in a nontrivial phase, see Fig. 3b, c, f, and g. Similar to group VB $M X_{2}$, the effects of halogen substitution and one-sided hydrogenation can be attributed to the addition of one electron in group VIIB $\mathrm{MX}_{2}$ films.

As already noted, in contrast to halogen substitution and onesided hydrogenation, pnictogen substitution results in a loss of one electron. Here, as shown in Fig. $3 \mathrm{~d}$ and $\mathrm{h}$, in the case of $2 \mathrm{H}$ $\mathrm{ReSeBr}$ one obtains a trivial insulator with $\mathrm{Z}_{2}=0$.
Clearly, the effects of pnictogen substitution, and halogen substitution or one-sided $\mathrm{H}$ adsorption are opposite for group VB and group VIIB in terms of the structural phase transitions and band topologies. Pnictogen substitution induces nontrivial phases for group VB but not for group VIIB. On the other hand, halogen substitution and one-sided $\mathrm{H}$ adsorption induces nontrivial phases for group VIIB but not for group VB. Notably, these nontrivial phases were only obtained when the structures undergo a phase transition from $2 \mathrm{H}$ to $1 \mathrm{~T}$ for both loss or gain of an electron.

Results for all other films considered, including their band structures, are given in Tables S1-S10 and Figs. S10-S24 in the Supplementary Materials.

In order to better understand the mechanism underlying the formation of the nontrivial phases, we carried out a detailed orbital analysis for one representative nontrivial film for each metal, as shown in Fig. S25. Since the GGA is well known to underestimate band gaps, we performed hybrid functional based calculations for nontrivial phases the selected films. We found that the key orbitas are the $\mathrm{M}-d_{y z}$ and $\mathrm{M}-d_{x z}$ orbitals for all selected cases as seen in the partial band projections in Fig. $4 a-f, h, k$. Without SOC, all selected systems are gapless in which the valence and conduction bands are degenerate at the $\Gamma$ point, and the systems are all metallic. With SOC, the doubly degenerate M- $d_{y z}$ $\mathrm{M}-d_{x z}$ orbitals experience a gap opening at the $\Gamma$ point in all these 

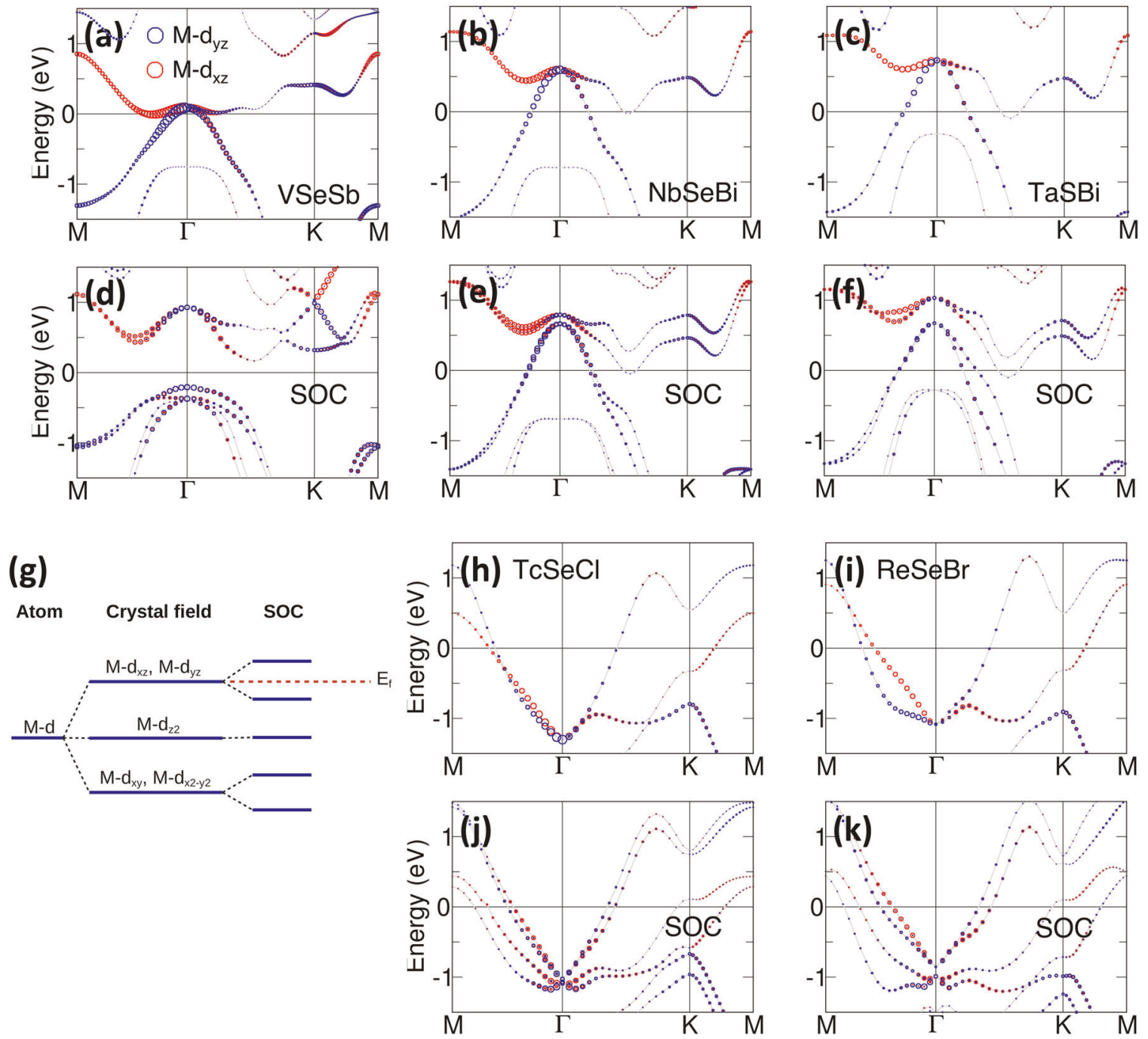

Fig. 4 Hybrid-functional based electronic band structures without SOC (top) and with SOC (bottom) of the selected stable structural phases associated with the $d_{y z}$ (blue dot) and $d_{x z}$ (red dot) orbitals. a, d $1 \mathrm{~T}$ VSeSb, b, e $1 \mathrm{~T} \mathrm{NbSeBi}, \mathbf{c}, \mathbf{f} 1 \mathrm{~T} \mathrm{TaSBi}, \mathbf{h}, \mathbf{j} 1 \mathrm{~T} \mathrm{TcSeCl}, \mathbf{i}, \mathbf{k} 1 \mathrm{~T} \mathrm{ReSeBr}$. g Schematic energly-level diagram highlighting how degeneracies of crystal-field split levels are lifted via SOC

selected films. In short, the $\mathrm{M}-d_{y z}$ and $\mathrm{M}-d_{x y}$ orbitals remain degenerate under the crystal-field, but this degeneracy is lifted via the effects of the SOC to open a gap at the Fermi energy at $\Gamma$ to drive the system into the topological phase, see Fig. $4 \mathrm{~g}$.

In terms of band gaps, surprisingly, 1T VSeSb becomes a topological insulator with a positive system-wide band gap (i.e. the minimum band gap throughout the Brillouin zone in the system around the Fermi energy) of $373 \mathrm{meV}$ and a band gap of $1135 \mathrm{meV}$ at the $\Gamma$ point. Although many other films we considered remained semi-metallic, we could still see a significant increase in the band gap at $\Gamma$ such as in the case of 1T TaSBi (with SOC) with a system-wide band gap of $360 \mathrm{meV}$, see Fig. 4f. Remarkably, the nontrivial phase is robust for selected groups of MXY films, demonstrating the likelihood of sustaining this nontrivial phase upon growing the film on a suitable substrate. It is noteworthy that the calculated band gaps of these materials are large enough for possible room temperature applications.

Figure $5 a$ and $b$ illustrates the topologically protected edge states in the real and reciprocal spaces of the predicted Janus 2D films in the quantum spin Hall (QSH) phases with the spin-up (blue) and spin-down (red) electrons conducting along one edge of the ribbon. Figure $5 \mathrm{c}$ shows a sheet of a TMD film with a region of Janus 2D to demonstrate the possibility of topological insulator phase upon substitutional doping. In our calculations, the topological insulator phase and several nontrivial phases are obtained in different compounds by directly replacing the top chalcogen with a certain dopant which has been discussed indepth in this study.

Finally, impressive experimental results ${ }^{50-57,59,60}$ have been reported from ultrathin pristine TMD, $\mathrm{VS}_{2}$, and $\mathrm{NbSe}_{2}$ films successfully synthesized via chemical vapor deposition (CVD). ${ }^{53,55}$ Several techniques have also been tested such as powder vapor deposition ${ }^{50}$ for $\mathrm{TaS}_{2}$, e-beam evaporation ${ }^{54}$ for $\mathrm{NbS}_{2}$, and modified Bridgman method ${ }^{60}$ for $\mathrm{ReS}_{2}$ and $\mathrm{ReSe}_{2}$ films. Notably, the Janus 2D material SMoSe ${ }^{35}$ has been successfully synthesized via controlled sulfurization. And very recently, a single-layer Bismuth (SLB) film has been successfully deposited on $\mathrm{NbSe}_{2}{ }^{52}$ These results suggest that viable Janus 2D NbSeBi films could be experimentally obtained via lithography or etching method. More generally, it will be interesting to explore the possibility of synthesizing the Janus 2D films from VB and VIIB elements by using the aforementioned methods and other mechanical exfoliation or bottom-up methods. 
(a)

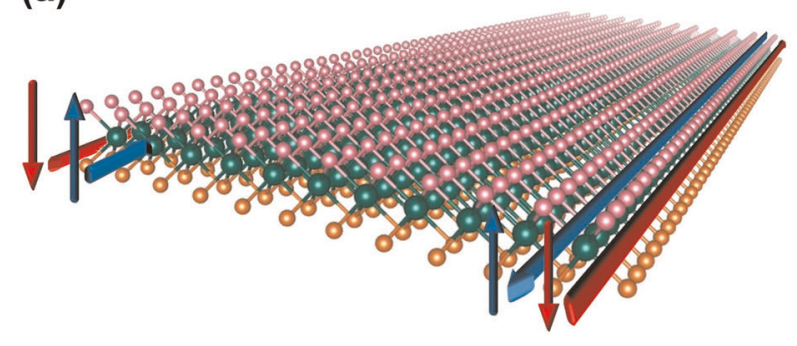

(b)

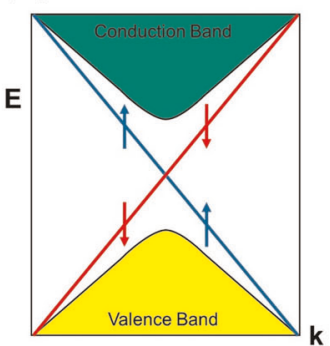

(c)

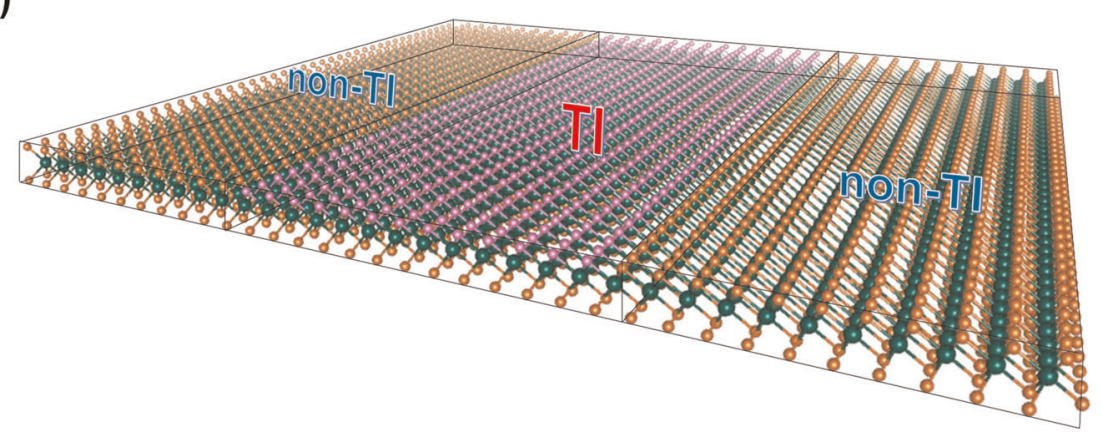

Fig. 5 a, b Illustrations of the topologically protected edge states, c Illustration of possible synthesis of Janus 2D materials via lithography methods

\section{METHODS}

The first-principles calculations were carried out using the Vienna Ab initio Simulation Package (VASP) ${ }^{63,64}$ with the projected augmented wave $(\mathrm{PAW})^{65}$ potentials. Spin polarization was considered in all cases for the comparison between the non-magnetic (NM) and ferromagnetic (FM) configurations. The exchange-correlation functional was treated within the Perdew-Burke-Ernzerhof (PBE) generalized gradient approximations (GGA). ${ }^{66-70}$ The cut-off energy used throughout the calculations was set to $400 \mathrm{eV}$. Atomic positions were optimized for each lattice constant value considered until the residual forces were no greater than $10^{-3} \mathrm{eV} / \mathrm{A}$. The criteria for energy convergence for self-consistency was set at $10^{-6} \mathrm{eV}$. The vacuum region along the $z$-direction was set to $\sim 15 \AA$ to prevent interactions between the repeated monolayer slabs under periodic boundary condition. A $\Gamma$-centered Monkhorst-Pack ${ }^{71}$ grid of $12 \times 12 \times 1$ in the first Brillouin zone was used for calculating atomic structures and lattice relaxations (pristine and substitutional TMDs). However, a denser grid of $36 \times 36 \times 1$ was used for band structure calculations in order to accurately capture band gaps and band topologies. The band topologies were further checked following the method of Refs. ${ }^{61,62}$ for calculating the Chern numbers and $Z_{2}$ topological invariants. The phonon spectra were calculated using the Phonopy code. ${ }^{72} \mathrm{~A} 2 \times 2 \times 1$ supercell with a grid of $36 \times 36 \times 1$ was constructed in order to obtain accurate force constants for calculating the phonon spectra. The molecular dynamics (MD) simulations were carried out in a $3 \times 3$ superlattice to determine the elevatedtemperature stability for $5000 \mathrm{fs}$ with a time step of $2.0 \mathrm{fs}$ at $T=500 \mathrm{~K}$.

\section{DATA AVAILABILITY}

All relevant data are included in the paper and/or its Supplementary Information files.

\section{CODE AVAILABILITY}

The calculations were implemented using the VASP, Wannier90, and Phonopy packages.

\section{ACKNOWLEDGEMENTS}

F.-C.C. acknowledges support from the National Center for Theoretical Sciences and the Ministry of Science and Technology of Taiwan under Grants Nos MOST-107-2628M-110-001-MY3. He is also grateful to the National Center for High-performance Computing for computer time and facilities. The work at Northeastern University was supported by the US Department of Energy (DOE), Office of Science, Basic Energy Sciences grant number DE-FG02-07ER46352 (core research), and benefited from Northeastern University's Advanced Scientific Computation Center (ASCC), the NERSC supercomputing center through DOE grant number DE-AC02-05CH11231, and support (applications to layered materials) from the DOE EFRC: Center for Complex Materials from First Principles (CCM) under DE-SC0012575.

\section{AUTHOR CONTRIBUTIONS}

F.-C.C. initiated the study. A.B.M., R.A.B.V., C.-H.H., Z.-Q.H., and L.-Y.F., performed firstprinciples calculations. A.B.M., R.A.B.V., C.-H.H., Z.-Q.H., E.F., H.L., A.B., and F.-C.C. performed analysis and contributed to discussions and wrote the manuscript.

\section{ADDITIONAL INFORMATION}

Supplementary information accompanies the paper on the npj 2D Materials and Applications website (https://doi.org/10.1038/s41699-019-0118-2).

Competing interests: The authors declare no competing interests.

Publisher's note Springer Nature remains neutral with regard to jurisdictional claims in published maps and institutional affiliations.

\section{REFERENCES}

1. Kane, C. L. \& Mele, E. J. Quantum spin hall effect in graphene. Phys. Rev. Lett. 95, 1-4 (2005).

2. Kane, C. L. \& Mele, E. J. $Z_{2}$ topological order and the quantum spin hall effect. Phys. Rev. Lett. 95, 3-6 (2005).

3. Hasan, M. Z. \& Kane, C. L. Colloquium: topological insulators. Rev. Mod. Phys. 82, 3045-3067 (2010).

4. Bansil, A., Lin, H. \& Das, T. Colloquium: topological band theory. Rev. Mod. Phys. 88, 1-37 (2016).

5. Kou, L., Ma, Y., Sun, Z., Heine, T. \& Chen, C. Two-Dimensional Topological Insulators: Progress and Prospects. J. Phys. Chem. Lett. 8, 1905-1919 (2017).

6. Liu, C.-C., Feng, W. \& Yao, Y. Quantum spin hall effect in silicene. Phys. Rev. Lett. 107, 076802 (2011).

7. Chuang, F. C. et al. Tunable topological electronic structures in Sb(111) bilayers: a first-principles study. Appl. Phys. Lett. 102, 1-5 (2013).

8. Xu, Y. et al. Large-gap quantum spin hall insulators in tin films. Phys. Rev. Lett. 111, 1-5 (2013). 
9. Chuang, F. C. et al. Prediction of large-gap two-dimensional topological insulators consisting of bilayers of group III elements with Bi. Nano Lett. 14, 2505-2508 (2014).

10. Fu, L. Topological crystalline insulators. Phys. Rev. Lett. 106, 1-4 (2011).

11. Hsieh, T. H. et al. Topological crystalline insulators in the SnTe material class. Nat Commun. 3, 982 (2012)

12. Wrasse, E. O. \& Schmidt, T. M. Prediction of two-dimensional topological crystalline insulator in PbSe monolayer. Nano Lett. 14, 5717-5720 (2014).

13. Hsu, C. H. et al. Two-dimensional topological crystalline insulator phase in $\mathrm{Sb} / \mathrm{Bi}$ planar honeycomb with tunable dirac gap. Sci. Rep. 6, 1-7 (2016).

14. Liu, C. C. et al. Low-energy effective Hamiltonian for giant-gap quantum spin Hall insulators in honeycomb X-hydride/halide $(\mathrm{X}=\mathrm{N}-\mathrm{Bi})$ monolayers. Phys. Rev. $B$ Condens. Matter Mater. Phys. 90, 195427 (2014).

15. Li, L., Zhang, X., Chen, X. \& Zhao, M. Giant topological nontrivial band gaps in chloridized gallium bismuthide. Nano Lett. 15, 1296-1301 (2015).

16. Crisostomo, C. P. et al. Robust large gap two-dimensional topological insulators in hydrogenated III-V buckled honeycombs. Nano Lett. 15, 6568-6574 (2015).

17. Yao, L. Z. et al. Predicted growth of two-dimensional topological insulator thin films of III-V compounds on Si(111) substrate. Sci. Rep. 5, 1-6 (2015).

18. Hsu, C. H. et al. The nontrivial electronic structure of $\mathrm{Bi} / \mathrm{Sb}$ honeycombs on $\mathrm{SiC}$ (0001). New J. Phys. 17, 025005 (2015).

19. Chuang, F. C. et al. Prediction of two-dimensional topological insulator by forming a surface alloy on Au/Si(111) substrate. Phys. Rev. B 93, 1-5 (2016).

20. Reis, F. et al. Bismuthene on a SiC substrate. Science 357, 287-290 (2017).

21. Hsu, C. H. et al. Growth of a predicted two-dimensional topological insulator based on InBi-Si(111)- $7 \times$ 7. Phys. Rev. B 98, 2-7 (2018).

22. Pierucci, D. et al. Tunable doping in hydrogenated single layered molybdenum disulfide. ACS Nano 11, 1755-1761 (2017).

23. Manzeli, S., Ovchinnikov, D., Pasquier, D., Yazyev, O. V. \& Kis, A. 2D transition metal dichalcogenides. Nat. Rev. Mater. 2, 17033 (2017).

24. Dolui, K., Rungger, I., Das Pemmaraju, C. \& Sanvito, S. Possible doping strategies for MoS2 monolayers: an ab initio study. Phys. Rev. B Condens. Matter Mater. Phys. 88, 1-9 (2013).

25. Qu, Y., Pan, H. \& Kwok, C. T. Hydrogenation-controlled phase transition on twodimensional transition metal dichalcogenides and their unique physical and catalytic properties. Sci. Rep. 6, 1-13 (2016).

26. Chen, W., Hou, X., Shi, X. \& Pan, H. Two-dimensional janus transition metal oxides and chalcogenides: multifunctional properties for photocatalysts, electronics, and energy conversion. ACS Appl. Mater. Interfaces 10, 35289-35295 (2018).

27. Onofrio, N., Guzman, D. \& Strachan, A. Novel doping alternatives for single-layer transition metal dichalcogenides. J. Appl. Phys. 122, 185102 (2017).

28. Raffone, F., Ataca, C., Grossman, J. C. \& Cicero, G. MoS 2 enhanced T-phase stabilization and tunability through alloying. J. Phys. Chem. Lett. 7, 2304-2309 (2016).

29. Suh, J. et al. Doping against the native propensity of $\mathrm{MoS}_{2}$ : degenerate hole doping by cation substitution. Nano Lett. 14, 6976-6982 (2014).

30. Zhang, K. et al. Manganese doping of monolayer $\mathrm{MoS}_{2}$ : the substrate is critical. Nano Lett. 15, 6586-6591 (2015).

31. Azcatl, A. et al. Covalent nitrogen doping and compressive strain in $\mathrm{MoS}_{2}$ by remote $\mathrm{N}_{2}$ plasma exposure. Nano Lett. 16, 5437-5443 (2016).

32. Zheng, Y. R. et al. Doping-induced structural phase transition in cobalt diselenide enables enhanced hydrogen evolution catalysis. Nat. Commun. 9, 2533 (2018).

33. Wang, Y. et al. Structural phase transition in monolayer $\mathrm{MoTe}_{2}$ driven by electrostatic doping. Nature 550, 487-491 (2017).

34. Liu, P. et al. Autoperforation of $2 \mathrm{D}$ materials for generating two-terminal memristive Janus particles. Nat. Mater. 17, 1005-1012 (2018).

35. Zhang, J. et al. Janus monolayer transition-metal dichalcogenides. ACS Nano 11, 8192-8198 (2017).

36. Ng, S. W., Noor, N. \& Zheng, Z. Graphene-based two-dimensional Janus materials. NPG Asia Mater. 10, 217-237 (2018).

37. Hsu, C. H. et al. Quantum anomalous Hall insulator phase in asymmetrically functionalized germanene. Phys. Rev. B 96, 1-6 (2017).

38. Crisostomo, C. P. et al. Chemically induced large-gap quantum anomalous Hall insulator states in III-Bi honeycombs. npjComput. Mater. 3, 39 (2017).

39. Jin, K. H. \& Jhi, S. H. Quantum anomalous hall and quantum spin-hall phases in flattened Bi and Sb bilayers. Sci. Rep. 5, 8426 (2015).

40. Hu, A. M., Wang, L. L., Xiao, W. Z., Xiao, G. \& Rong, Q. Y. Electronic structures and magnetic properties in nonmetallic element substituted $\mathrm{MoS}_{2}$ monolayer. Comput. Mater. Sci. 107, 72-78 (2015).

41. Cheng, Y. C., Zhu, Z. Y., Mi, W. B., Guo, Z. B. \& Schwingenschlögl, U. Prediction of two-dimensional diluted magnetic semiconductors: doped monolayer $\mathrm{MoS}_{2}$ systems. Phys. Rev. B Condens. Matter Mater. Phys. 87, 2-5 (2013).

42. Suh, J. et al. Reconfiguring crystal and electronic structures of $\mathrm{MoS}_{2}$ by substitutional doping. Nat. Commun. 9, 199 (2018).
43. Yue, Q., Chang, S., Qin, S. \& Li, J. Functionalization of monolayer $\mathrm{MoS}_{2}$ by substitutional doping: a first-principles study. Phys. Lett. A Gen. Solid State Phys. 377, 1362-1367 (2013).

44. Brown, P. A., Xu, C. \& Shuford, K. L. Periodic trends of pnictogen substitution into a graphene monovacancy: a first-principles investigation. Chem. Mater. 26, 5735 (2014).

45. Guo, S., Wang, Y., Wang, C., Tang, Z. \& Zhang, J. Large spin-orbit splitting in the conduction band of halogen $\left(\mathrm{F}, \mathrm{Cl}, \mathrm{Br}\right.$, and I) doped monolayer $\mathrm{WS}_{2}$ with spinorbit coupling. Phys. Rev. B 96, 1-7 (2017).

46. Rasmussen, F. A. \& Thygesen, K. S. Computational 2D materials database: electronic structure of transition-metal dichalcogenides and oxides. J. Phys. Chem. C 119, 13169-13183 (2015).

47. Lebègue, S., Björkman, T., Klintenberg, M., Nieminen, R. M. \& Eriksson, O. Twodimensional materials from data filtering and Ab Initio calculations. Phys. Rev. X 3 1-7 (2013).

48. Ataca, C., Şahin, H. \& Ciraci, S. Stable, single layer $M X_{2}$ 2012. J. Phys. Chem. C 116, 8983-8999 (2012).

49. Hart, L. S. et al. Electronic bandstructure and van der Waals coupling of ReSe2revealed by high-resolution angle-resolved photoemission spectroscopy. Sci. Rep. 7, 1-9 (2017).

50. Zhao, R. et al. Two-dimensional tantalum disulfide: controlling structure and properties via synthesis. 2D Mater. 5, 025001 (2018).

51. Hajiyev, P., Cong, C., Qiu, C. \& Yu, T. Contrast and Raman spectroscopy study of single- and few-layered charge density wave material: 2H-TaSe2. Sci. Rep. 3, 2593 (2013)

52. Fang, A. et al. Bursting at the seams: rippled monolayer bismuth on $\mathrm{NbSe}_{2}$. Sci. Adv. 4, eaaq0330 (2018).

53. Wang, $\mathrm{H}$. et al. High-quality monolayer superconductor $\mathrm{NbSe}_{2}$ grown by chemical vapour deposition. Nat. Commun. 8, 394 (2017).

54. Bark, H. et al. Large-area niobium disulfide thin films as transparent electrodes for devices based on two-dimensional materials. Nanoscale 10, 1056-1062 (2018).

55. Ji, Q. et al. Metallic vanadium disulfide nanosheets as a platform material for multifunctional electrode applications. Nano Lett. 17, 4908-4916 (2017).

56. Boscher, N. D., Blackman, C. S., Carmalt, C. J., Parkin, I. P. \& Prieto, A. G. Atmospheric pressure chemical vapour deposition of vanadium diselenide thin films. Appl. Surf. Sci. 253, 6041-6046 (2007).

57. Wang, Y., Sofer, Z., Luxa, J. \& Pumera, M. Lithium exfoliated vanadium dichalcogenides $\left(\mathrm{VS}_{2}, \mathrm{VSe}_{2}, \mathrm{VTe}_{2}\right.$ ) exhibit dramatically different properties from their bulk counterparts. Adv. Mater. Interfaces 3, 1-8 (2016).

58. Tongay, S. et al. Monolayer behaviour in bulk $\operatorname{ReS}_{2}$ due to electronic and vibrational decoupling. Nat. Commun. 5, 1-6 (2014).

59. Chenet, D. A. et al. In-plane anisotropy in mono- and few-layer $\operatorname{ReS}_{2}$ probed by Raman spectroscopy and scanning transmission electron microscopy. Nano Lett. 15, 5667-5672 (2015).

60. Jariwala, B. et al. Synthesis and characterization of $\mathrm{ReS}_{2}$ and $\mathrm{ReSe}_{2}$ layered chalcogenide single crystals. Chem. Mater. 28, 3352-3359 (2016).

61. Fukui, T. \& Hatsugai, Y. Quantum spin Hall effect in three dimensional materials: Lattice computation of $\mathrm{Z}_{2}$ topological invariants and its application to $\mathrm{Bi}$ and $\mathrm{Sb}$. J. Phys. Soc. Jpn. 76, 1-5 (2007).

62. Qiao, Z. et al. Quantum anomalous Hall effect in graphene from Rashba and exchange effects. Phys. Rev. B Condens. Matter Mater. Phys. 82, 3-6 (2010).

63. Kresse, G. \& Hafner, J. Ab initio molecular dynamics for liquid metals. Phys. Rev. $B$ 47, 558-561 (1993)

64. Kresse, G. \& Furthmu, J. Efficient iterative schemes for ab initio total-energy calculations using a plane-wave basis set. Phys. Rev. B 54, 11169 (1996).

65. Joubert, D. From ultrasoft pseudopotentials to the projector augmented-wave method. Phys. Rev. B Condens. Matter Mater. Phys. 59, 1758-1775 (1999).

66. Rajagopal, A. K. \& Callaway, J. Inhomogeneous electron gas. Phys. Rev. B 7, 1912-1919 (1973).

67. Kohn, W. \& Sham, L. J. Self-consistent equations including exchange and correlation effects. Phys. Rev. 140, A1133 (1965).

68. Ceperley, D. M. \& Alder, B. J. Ground state of the electron gas by a stochastic model. Phys. Rev. Lett. 45, 566-569 (1980).

69. Perdew, J. P. \& Zunger, A. Self-interaction correction to density-functional approximations for many-electron systems. Phys. Rev. B 23, 5048-5079 (1981).

70. Perdew, J. P., Burke, K. \& Ernzerhof, M. Generalized gradient approximation made simple. Phys. Rev. Lett. 77, 3865-3868 (1996).

71. Pack, J. D. \& Monkhorst, H. J. 'Special points for Brillouin-zone integrations'-a reply. Phys. Rev. B 16, 1748-1749 (1977).

72. Tanaka, I. Scripta materialia first principles phonon calculations in materials science. Scr. Mater. 108, 1-5 (2015). 
Open Access This article is licensed under a Creative Commons Attribution 4.0 International License, which permits use, sharing, adaptation, distribution and reproduction in any medium or format, as long as you give appropriate credit to the original author(s) and the source, provide a link to the Creative Commons license, and indicate if changes were made. The images or other third party material in this article are included in the article's Creative Commons license, unless indicated otherwise in a credit line to the material. If material is not included in the article's Creative Commons license and your intended use is not permitted by statutory regulation or exceeds the permitted use, you will need to obtain permission directly from the copyright holder. To view a copy of this license, visit http://creativecommons. org/licenses/by/4.0/.

(c) The Author(s) 2019 